\title{
EL OSTIÓN DEL NORTE CHILENO (Argopecten Purpuratus), UN ALIMENTO DE ALTO VALOR NUTRICIONAL
}

\section{THE SCALLOP FROM THE CHILEAN NORTH (Argopecten Purpuratus), A FOOD WITH A HIGH NUTRITIONAL VALUE}

\author{
Alfonso Valenzuela B. (1), Carmen Gloria Yánez (2), Constanza Golusda V. (2) \\ (1) Centro de Lípidos \\ (2) Dirección de Asistencia Técnica, \\ Instituto de Nutrición y Tecnología de los Alimentos (INTA), Universidad de Chile. Santiago, Chile.
}

\begin{abstract}
Mollusks are good food and also products of great commercial value. However, information about the nutritional value of these shellfish as foods is scarce. This work focused on the chemical characterization of scallops, one of the main products of the Chilean aquaculture. Samples of frozen scallops from three culture centers located in the north of Chile (Coquimbo Region) were subjected to several chemical analysis to obtain information about their nutritional composition. Results show that per serving (approximately $100 \mathrm{~g}$ ) scallops provide low fat, but containing an interesting amount of omega-3 fatty acids (EPA and DHA). Carbohydrate and cholesterol content are also low. Phytosterol content of scallops comprises $30 \%$ of the total sterols, presumable derived from their feeding. Scallops are also a good supply of tryptophan, vitamin B12 and minerals. For these reasons scallops are very good nutritional food.
\end{abstract}

Key words: scallops, chemical composition, nutritional value.

Este trabajo fue recibido el 24 de Agosto de 2010 y aceptado para ser publicado el 5 de Mayo de 2011.

\section{INTRODUCCIÓN}

La acuicultura en Chile se ha transformado, en las últimas décadas, en una importante actividad económica. Destaca, sin lugar a dudas, la acuicultura del salmón por sus altos volúmenes de producción y relevancia internacional (1). Sin embargo, existen otras actividades acuicultoras, principalmente de moluscos bivalvos, como es el caso de los choritos, las ostras y el ostión, entre los más importantes (2). Cada uno de ellos tiene características particulares desde el punto de vista organoléptico y sensorial, lo que les ha permitido tener una demanda creciente en los diferentes mercados internacionales. Sin embargo, poco es lo que se ha realizado para valorizar desde el punto de vista nutricional a estos moluscos como alimentos, ya que habitualmente se les considera como productos gourmet de alta demanda, sin considerar sus posibles atributos nutricionales. Este trabajo desarrolló la caracterización de la composición química de uno de estos productos de la acuicultura chilena: el ostión (Argopecten purpuratus)
El ostión que se cultiva en el norte chileno es un molusco bivalvo de reconocida demanda en el mercado internacional. Se consume principalmente frescorefrigerado y congelado. El 95\% de la producción se exporta y solo un $5 \%$ se destina al consumo interno. Los principales destinos de exportación son los países europeos, siendo Francia el mayor consumidor (87\% del volumen exportado), seguido de Italia $(6,5 \%)$ y España $(3,3 \%)$ (2). El monto total de las exportaciones en 2008 fue de 2.922 toneladas lo que significó un ingreso de US\$ 33,2 millones (3).

La producción nacional se concentra principalmente en las Regiones de Atacama (28\%) y Coquimbo $(70 \%)$, las que representan en conjunto el $75 \%$ de las exportaciones totales. El cultivo del ostión representa una importante actividad económica en estas regiones, siendo la Región de Coquimbo la que concentra la mayor actividad ya que en ella el ostión es el principal, o único producto, particularmente en la Bahía de Tongoy donde genera más de 1.600 empleos directos (4). La producción 
nacional no está exenta de competencia, siendo Perú, y en menor medida los propios países europeos y algunos países asiáticos, los principales oferentes con precios muy competitivos.

El ostión, y los mariscos en general, son muy apreciados por su sabor, palatabilidad, apariencia y más secundariamente por algunos mitos sobre sus supuestos poderes "afrodisíacos", no siendo productos de gran consumo en Chile. Por este motivo, la investigación sobre las propiedades nutricionales de los productos del mar se ha centrado principalmente en los peces, siendo más escasa la información sobre los mariscos y en particular sobre los moluscos. Sin embargo, la reducción de la captura mundial de peces, el aumento de los precios de los productos y sub-productos (harina y aceite, principalmente) y las recomendaciones de limitar su consumo en algunos grupos etarios (embarazadas y alérgicos, entre otros) ha llevado a que el consumo de pescado a nivel mundial se reduzca. En nuestro país el consumo no es superior a 5-6 kg año/cápita (1), muy por debajo de las recomendaciones (5). Debido a esto, hoy en día se valoriza cada vez más a los mariscos como una alternativa nutricional de buena calidad y cada vez mayor disponibilidad debido a la creciente actividad de cultivo de algunos de estos animales. Dentro de la variedad de mariscos, y particularmente de los moluscos, que se cultivan en Chile, el ostión es uno de los más importantes.

Las partes blandas comestibles del ostión la constituyen el músculo abductor (o "tallo") y las gónadas (también llamadas "coral"), con lo cual desde el punto de vista de su consumo se identifican como "ostión con coral" y "ostión sin coral", lo que determina una composición química y un valor nutricional diferente. A continuación se describen los resultados del estudio de la composición química de muestras de ostiones con coral y sin coral obtenidas desde tres centros de cultivo de la Región de Coquimbo, ubicados en las localidades de Guanaqueros (Pesquera Camanchaca), Bahía Inglesa (Pesquera Camanchaca) y Tongoy (Invertec).

\section{MATERIALES Y MÉTODOS}

\section{Obtención y caracterización de las muestras}

Las muestras de ostiones, provenientes de los diferentes centros de cultivo, fueron extraídas por los operadores de cada centro obteniéndose el músculo abductor junto con las gónadas, producto que se identifica como "ostión con coral" y solamente el músculo abductor, producto que se identifica como "ostión sin coral". La obtención de las muestras fue supervisada por personal de la Asociación de Productores de Ostras y Ostiones de Chile (APOOCH). Las muestras de ostiones con y sin coral provenientes de la localidad de Guanaqueros, fueron identificadas con la letra "G". La muestras provenientes de Bahía Inglesa se identificaron como "B" y las de Tongoy como "T". Se obtuvo un total de veinte muestras de aproximadamente $2 \mathrm{~kg}$ c/u. Las muestras, previamente congeladas, se enviaron por vía aérea a Santiago y fueron recibidas en el Centro de Alimentos del Instituto de Nutrición y Tecnología de los Alimentos (INTA).

\section{Procedimientos analíticos}

Las muestras fueron homogenizada en frío mediante una licuadora y distribuidas a los diferentes laboratorios que participaron en el estudio (Centro de Lípidos, Centro de Alimentos y Laboratorio de Cromatografía) para la realización de los siguientes análisis: análisis proximal (6), perfil de ácidos grasos mediante cromatografía gaseosa capilar (7), contenido de colesterol mediante cromatografía gaseosa capilar (8), contenido de fitoesteroles mediante cromatografía gaseosa capilar (9), contenido de calcio, fósforo, magnesio, potasio, y sodio por espectrometría de absorción atómica (10), vitamina A mediante técnica HPLC (11), vitamina C mediante técnica HPLC (12), vitamina D mediante técnica HPLC (13) y vitamina E mediante técnica HPLC (14). Los resultados se expresaron como promedio \pm D.E. para un "n" de 2 a 5 muestras sometidas a análisis. Para el análisis estadístico se aplicó ANOVA seguido de test de Newman-Keuls, para lo cual un $p<0,5$ se consideró estadísticamente significativo.

\section{RESULTADOS}

La tabla 1 (A) y (B) muestra los resultados del análisis proximal de las muestras de ostiones con coral y sin coral, respectivamente. En términos generales, no hay diferencias entre las muestras provenientes de los diferentes lugares de recolección. Las pequeñas diferencias que fue posible observar en algunos valores, se pueden deber al tipo de alimentación de los ejemplares, la que estaría determinada por el diferente lugar de origen de estos.

La tabla 2 (A) y (B) muestra el contenido de ácido eicosapentaenoico (20:5, EPA), de ácido docosahexaenoico (22:6, DHA) y de ácidos grasos omega-3 totales (incluye el ácido docosapentaenoico, 22:5, DPA) obtenidos del perfil de ácidos grasos de las muestras de ostiones con coral y sin coral, respectivamente. Es interesante destacar que el ostión con coral contienen más EPA que DHA. Por el contrario, el ostión sin coral, aunque tiene menor cantidad de ambos ácidos grasos omega-3 por unidad de peso, contiene más DHA que EPA. 
La tabla 3 (A) y (B) muestra el contenido de esteroles (colesterol, $\beta$-sitosterol, campesterol y stigmasterol) de las muestras de ostiones con coral y sin coral, respectivamente. En ambos tipos de muestras, destaca el alto contenido de fitoesteroles, un componente habitualmente no evaluado en muestras de animales marinos.
La tabla 4 (A) y (B) muestra el contenido de minerales de las muestras de ostiones con coral y sin coral, respectivamente.

La tabla 5 (A) y (B) muestra el contenido de vitaminas hidrosolubles (C) y liposolubles (A, D y E) de las muestras de ostiones con coral y sin coral, respectivamente.

\section{TABLA 1}

Análisis proximal de ostiones (g/100 g)

\section{(A) con coral}

Muestra

Humedad

Cenizas

Proteína Grasa total

Hidratos de carbono

Energía (Kcal/100g)

$\mathrm{G} n=2$ $80,19 \pm 0,19$

$1,70 \pm 0,30$

$15,64 \pm 0,24$

$1,35 \pm 0,12$

$1,14 \pm 0,04$

79,0

$\mathrm{B} n=3$

$80,56 \pm 0,13$

$1,34 \pm 0,12$

$15,44 \pm 0,60$

$1,30 \pm 0,09$

$1,35 \pm 0,09$

79,0

$\mathrm{T} \mathrm{n}=5$

$81,18 \pm 0,14$

$1,08 \pm 0,90$

$15,37 \pm 1,98$

$1,21 \pm 0,16$

$1,17 \pm 0,31$

77,0

Promedio

$80,64 \pm 0,15$

$1,37 \pm 0,44$

$15,48 \pm 1,60$

$1,29 \pm 0,12$

$1,22 \pm 0,14$

78,0

\section{(B) sin coral}

Muestra

Humedad Cenizas Proteína Grasa total
Hidratos
de carbono

Energía

(Kcal/100g)

$\mathrm{G} \mathrm{n}=3$

\section{$79,27 \pm 0,36$}

$1,38 \pm 0,03$

$15,19 \pm 0,75$

$0,55 \pm 0,14$

$3,62 \pm 0,55$

80,0

$\mathrm{B} n=2$

$80,17 \pm 0,04$

$1,60 \pm 0,17$

$15,17 \pm 0,10$

$0,61 \pm 0,06$

$2,46 \pm 0,03$

76,0

$\mathrm{T} \mathrm{n}=5$

$80,64 \pm 0,36$

$1,20 \pm 0,29$

$15,56 \pm 0,38$

$0,54 \pm 0,05$

$2,07 \pm 0,60$

75,0

Promedio

$80,02 \pm 0,25$

$1,39 \pm 0,25$

$15,31 \pm 0,41$

$0,57 \pm 0,08$ *

$2,72 \pm 0,39 *$

77,0

* Valores significativamente diferentes $(\mathrm{p}<0,5)$ en comparación con ostiones con coral.

\section{TABLA 2}

\section{Contenido de ácidos grasos omega-3 de ostiones (mg/100 g)}

\section{(A) con coral}

\section{Muestra}

$\mathrm{G} n=2$

$\mathrm{B} n=3$

$\mathrm{T} n=5$

Promedio

(B) sin coral

Muestra

$\mathrm{G} n=3$

$\mathrm{B} n=2$

$\mathrm{T} n=5$

Promedio
EPA

$230 \pm 10$
$236 \pm 26$
$228 \pm 15$
$234 \pm 17$

EPA

$103 \pm 3$

$107 \pm 3$

$100 \pm 12$

$103 \pm 7^{*}$

\section{DHA}

$152 \pm 5,0$

$189 \pm 20$

$134 \pm 20$

$158 \pm 15$

\section{Omega -3 totales}

$430 \pm 17$

$458 \pm 57$

$388 \pm 38$

$425 \pm 37$

* Valores significativamente diferentes $(\mathrm{p}<0,5)$ en comparación con ostiones con coral. 


\section{DISCUSIÓN}

El análisis proximal del ostión con y sin coral, cuyos resultados se presentan en la tabla 1 (A y B) muestra un contenido proteico relativamente alto $(15 \mathrm{~g} / 100 \mathrm{~g})$ y un muy bajo contenido de grasa, especialmente en el ostión sin coral $(0,57 \mathrm{~g} / 100 \mathrm{~g})$. Esta grasa tiene una composición interesante, por su alto contenido de ácidos grasos omega-3, principalmente EPA y DHA, cuyos valores se muestran en la tabla 2 (A) y (B). El consumo de ambos ácidos grasos tiene efectos muy positivos en la salud cardiovascular y del sistema nervioso. Es ampliamente reconocido el efecto del EPA como hipotrigliceridémico, antiinflamatorio, hipotensor, antiarrítmico e hipocolesterolémico (15). El DHA se caracteriza por sus efectos positivos en la función del sistema nervioso y visual (16) y en la protección al desarrollo de enfermedades neurológicas, como Alzheimer (17), Parkinson (18) y patologías asociadas al comportamiento (19).

De acuerdo a la información obtenida, el consumo de 100 g (5 a 8 unidades) de ostión con coral, aportaría más del doble (425 mg) de ácidos grasos omega-3 que la recomendación de consumo diario (200 mg) de estos

\section{TABLA 3}

\section{Contenido de esteroles de ostiones (mg/100 g)}

\section{(A) con coral}

Muestra

$\mathrm{G} n=2$

$\mathrm{B} n=3$

$\mathrm{T} \mathrm{n}=5$

Promedio

Colesterol
$55,90 \pm 3,20$
$58,13 \pm 3,51$
$60,59 \pm 4,78$
$58,20 \pm 3,92$

(B) sin coral

Muestra

$\mathrm{G} n=3$

$\mathrm{B} n=2$

$\mathrm{T} n=5$

Promedio
Colesterol

$47,49 \pm 4,09$

$48,44 \pm 1,90$

$52,63 \pm 7,48$

$49,52 \pm 6,38$

\section{及 - Sitosterol}

$4,93 \pm 0,58$

$6,74 \pm 0,43$

$6,08 \pm 0,55$

$5,92 \pm 0,56$

$\boldsymbol{\beta}$ - Sitosterol
$7,82 \pm 2,35$
$6,13 \pm 1,90$
$6,75 \pm 1,39$
$6,90 \pm 1,52$

Campesterol

0,00

0,00

0,00

0,00

Campesterol

$5,49 \pm 1,72$

0,00

$2,96 \pm 3,13$

$2,81 \pm 3,08$
Stigmasterol

$15,46 \pm 2,56$

$19,06 \pm 2,24$

$18,36 \pm 4,39$

$17,62 \pm 3,95$

\section{TABLA 4}

Contenido de minerales de ostiones (mg/100 g)

\section{(A) con coral}

\section{Muestra}

$\mathrm{G} n=2$

$\mathrm{B} n=3$

$\mathrm{T} \mathrm{n}=5$

Promedio

\section{Calcio}

$23,90 \pm 2,20$

$26,30 \pm 1,35$

$24,10 \pm 1,14$

$24,80 \pm 1,22$

\section{Fósforo}

$116,0 \pm 12,0$

$154,0 \pm 4,0$

$148,0 \pm 6,80$

$156,0 \pm 7,30$
Magnesio

$38,70 \pm 7,00$

$38,80 \pm 3,00$

$41,40 \pm 8,20$

$39,60 \pm 7,92$

Fósforo

$135,0 \pm 5,60$

$127,0 \pm 0,3$

$126,0 \pm 2,00$

$129,0 \pm 2,18 *$
Magnesio

$40,06 \pm 2,00$

$38,00 \pm 0,20$

$36,20 \pm 3,00$

$38,30 \pm 2,11$
Potasio

$238,5 \pm 16,0$

$257,0 \pm 11,0$

$244,2 \pm 6,20$

$261,0 \pm 7,12$
Stigmasterol

$16,26 \pm 1,57$

$14,80 \pm 1,80$

$16,12 \pm 2,79$

$15,73 \pm 2,61$
$\mathrm{T} \mathrm{n}=5$

Promedio
$24,00 \pm 1,40$

$25,30 \pm 1,49$

* Valores significativamente diferentes $(\mathrm{p}<0,5)$ en comparación con ostiones con coral 
importantes nutrientes (20), aspecto que resulta muy positivo, ya que el ostión podría ser una manera "sabrosa" de consumir ácidos grasos omega-3 de origen marino.

Generalmente, en los alimentos de origen animal lo que se expresa como resultado analítico del contenido de esteroles, es el colesterol, sobre todo cuando se utilizan técnicas colorimétricas. Sin embargo, lo que realmente se evalúa son esteroles totales, entre los cuales no sólo está el colesterol, sino diferentes fitoesteroles, esto es esteroles de origen vegetal. La tabla 3 muestra el contenido de colesterol y el contenido de los fitoesteroles b-sitosterol, campesterol y stigmasterol, obtenidos de las muestras de ostión analizadas. Habitualmente se considera que el consumo de mariscos aporta altos contenidos de colesterol, lo cual es cierto en algunos de ellos, pero no en el caso del ostión con o sin coral. Una porción de ostiones con coral (5 a 8 unidades, $100 \mathrm{~g}$ ) aporta, en promedio, $58 \mathrm{mg}$ de colesterol, una cantidad equivalente a la aportada por una porción similar $(100 \mathrm{~g})$ de trucha o salmón (57 mg). Un huevo (50 g en promedio) aporta en promedio $300 \mathrm{mg}$ de colesterol. Más destacable es el alto contenido de fitoesteroles, ya que en el ostión con coral constituyen el $29 \%$ del total de esteroles y en el ostión sin coral el 34\%. Los fitoesteroles son reconocidos productos naturales con efectos hipocolesterolémicos (21), esto es su consumo inhibe la absorción del colesterol en el tubo digestivo (22). Se podría argumentar, entonces, que el consumo de ostiones podría tener un efecto colesterolémico menor, debido al consumo simultáneo de fitoesteroles. Se estima que el $50 \%$ de colesterol dietario y endógeno se reabsorbe en el intestino (23).
La presencia de fitoesteroles podría reducir esta reabsorción. La figura 1 muestra un cuadro comparativo del aporte de colesterol de diferentes alimentos, donde se puede observar que el ostión ocupa un lugar de menor importancia entre los alimentos incorporados a la figura que aportan colesterol.

Otra característica nutricional del ostión es su proteína que tiene un alto contenido del aminoácido esencial triptófano. Aunque no se realizó el análisis de la composición aminoacídica de la proteína del ostión, la literatura indica que una porción de ostión (5 a 8 unidades) aporta $260 \mathrm{mg}$ de triptófano que es equivalente al $81 \%$ del requerimiento diario de este importante aminoácido esencial (24). El bajo aporte de carbohidratos (1,2 g/100 g para el ostión con coral y 2,7 g/100 g para el ostión sin coral) lo transforma en un alimento adecuado para aquellos individuos con trastornos en el metabolismo de carbohidratos, lo cual es valioso debido a las altas tasas de diabetes mellitus que actualmente presenta la población. El ostión es también una muy buena fuente de minerales y de algunas vitaminas. El aporte de fósforo, magnesio y de potasio es interesante. Una porción de ostiones puede aportar hasta el $20 \%$ del requerimiento diario de fósforo, un $19 \%$ del requerimiento diario de magnesio y el $12 \%$ del requerimiento diario de potasio (24). El magnesio y el potasio, están relacionados con el control de homeostasis vascular, el equilibrio hidrosalino y la función del sistema nervioso y muscular (25).

El aporte de vitaminas A, C, D y E del ostión no resulta relevante del punto de vista nutricional. La literatura comunica que el ostión constituye una buena

\section{TABLA 5}

\section{Contenido de vitaminas de ostiones (cantidad en 100 g)}

(A) con coral Muestra

$\mathrm{G} n=2$

$\mathrm{B} \mathrm{n}=3$

$\mathrm{T} n=5$

Promedio
Vit. A (ug ER)*

1,23

ND

$2,96 \pm 1,44$

No estimable
Vit. C (mg)

ND

ND

ND

ND
Vit. D (mg)

$$
0,55
$$

$0,36 \pm 0,40$

$0,90 \pm 0,11$

0,060
Vit. E (mg ET)**

ND

ND

ND

ND

(B) sin coral

\section{Muestra}

$\mathrm{G} n=2$

$\mathrm{B} \mathrm{n}=3$

$\mathrm{T} n=5$

Promedio
Vit. A (ug ER)*

0,74

ND

$2,96 \pm 1,44$

No estimable

Vit. C (mg)
ND
ND
ND

Equivalente retinol

** Equivalente $\alpha$-tocoferol

ND: No detectado 
fuente de vitamina B12 (cianocobalamina). Aunque en este trabajo no se determinó el contenido de esta vitamina, la literatura indica que una porción de ostiones puede aportar 2 ug de cianocobalamina (26) lo que equivale al $33 \%$ del requerimiento diario de vitamina B12 (27). La vitamina B12 es un importante protector de la salud cardiovascular (28) ya que es necesaria para la metabolización de la homocisteína a metabolitos no dañinos (29).

De esta forma, a los conocidos atributos organolépticos del ostión, que lo hace un producto gourmet muy apreciado, es necesario agregar que se trata de un alimento muy saludable. Aunque su contenido de lípidos es muy bajo, aporta una interesante cantidad de ácidos grasos omega-3 EPA y DHA, no constituye un aporte importante de colesterol, más aún, sí es un aporte de fitoesteroles, agentes hipocolesterolémicos de origen natural (30). Su proteína aporta triptófano y su contenido de carbohidratos es bajo, con lo cual se trataría de un alimento de bajo impacto glicémico. Es una buena fuente de fosfato, magnesio y de potasio. Aunque su aporte de vitaminas liposolubles es bajo, destaca su aporte de vitamina B12. En resumen, el ostión, además de sus conocidos atributos organolépticos sería también un alimento de buen valor nutricional.

\section{RESUMEN}

Los moluscos, además de constituir un alimento, son productos de gran valor comercial. Sin embargo, es poco es lo que se hecho para valorizar desde el punto de vista nutricional a estos mariscos como alimentos. Este trabajo desarrolla la caracterización de la composición química del ostión, uno de los principales mariscos de la acuicultura chilena. Muestras de ostión congeladas provenientes de tres centros de cultivo del norte chileno (Región de Coquimbo) se sometieron a diferentes análisis con la finalidad de conocer su composición química. Los análisis muestran que una porción de ostiones (100 g aproximadamente) aporta poca grasa, pero contiene una interesante cantidad de ácidos grasos omega-3 (EPA y DHA), además presenta un bajo contenido de carbohidratos y de colesterol. Un aspecto novedoso es el contenido de fitoesteroles de los ostiones, derivado de su alimentación, los que son en promedio el $30 \%$ del contenido total de esteroles. El ostión, además, constituye un buen aporte de triptófano, de vitamina B12 (cobalamina) y de sales minerales. Por este motivo el consumo del ostión, además de ser placentero, es un muy buen alimento.

Palabras clave: ostiones, composición química, valor nutricional

\section{FIGURA 1}

Contenido de colesterol de algunos alimentos

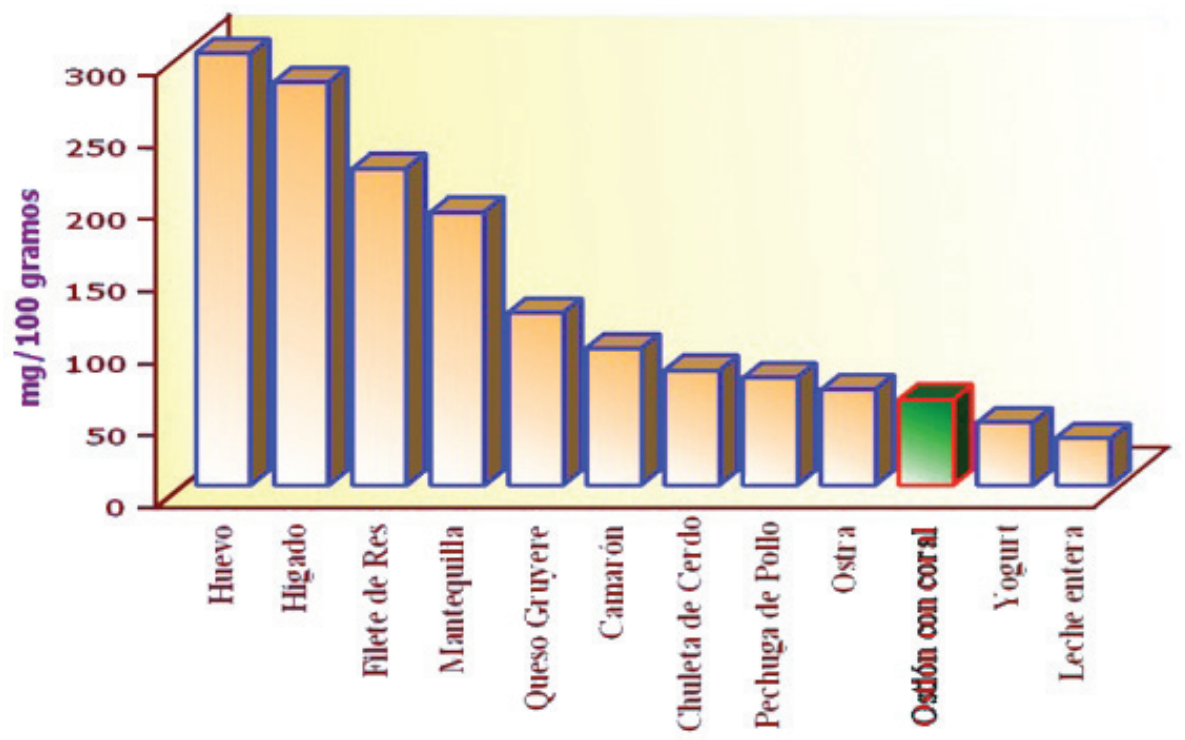


Dirigir la correspondencia a:

Profesor

Alfonso Valenzuela B.

Centro de Lípidos

Instituto de Nutrición y Tecnología de los Alimentos

(INTA)

Universidad de Chile, Santiago, Chile

Teléfono: 9781449

Fax: 2214030

E-mail:avalenzu@inta.uchile.cl

Los autores declaran no tener conflicto de intereses con las entidades que financiaron y patrocinaron el desarrollo de la investigación.

Agradecimientos: El estudio analítico fue financiado por el Gobierno Regional de la Región de Coquimbo y patrocinado por la Asociación de Productores de Ostras y Ostiones de Chile (APOOCH), a quienes agradecemos.

\section{BIBLIOGRAFÍA}

1. Valenzuela, A. El Salmón: un banquete de salud. Rev Chil Nutr. 2005; 32: 8-17.

2. Araneda, D. El consumo de productos pesqueros y de acuicultura en Chile. Ministerio de Economía, Fomento y Reconstrucción. Informe Técnico N 43, 2009.

3. Informe Sectorial de Pesca y Acuicultura, Subsecretaría de Pesca, Gobierno de Chile, noviembre 2010.

4. Asociación de Productores de Ostras y Ostiones de Chile (APOOCH), informe de actividad anual 2009.

5. Wolmarans, P. Background paper on global trends in food production, intake and composition. Ann Nutr Metab 2009; 55: 244-72.

6. Manual de Métodos de Análisis Fisicoquímico de Alimentos, Aguas y Suelos. Ministerio de Salud Pública. Ediciones Instituto de Salud Pública, 1998.

7. Morrison, W., Smith, L. Preparation of fatty acid methyl esther and dimethylacetals from lipids with fluoride - methanol. J Lipid Res 1964; 25: 600-608.

8. "Cholesterol in Foods". Método Oficial AOAC 994.10. AOAC Pub. 2001.

9. Sorenson, W., Sullivan, D. Determination of phytosterols and sterols by gas-liquid chromatography. J AOAC Int. 2006; 34: 123-8.

10. Mattleson, P. Manual de metodologías para la determinación de metales y minerales mediante espectrofotometría de absorción atómica. Edición de Manuales Analíticos, Perkin-Elmer de México, México, DF, 2002.

11. Diplock, A., Green, J., Bunyan, J. The determination of vitamin $\mathrm{A}$ in animal tissues and its presence in the liver of the vitamin A-deficient rat. Brit J Nutr 1983; 27: 199-204.

12. Fontannaz, P., Kilinc, T., Heudi, O. HPLC-UV determination of total vitamin $\mathrm{C}$ in a wide range of fortified food products. Food Chem. 2006; 94: 626-31.

13. Determinación de vitamina D3 en alimentos y premezclas por HPLC con detector UV. Métodos AOAC 981.17 y 982.29, AOAC, 16 Ed., vol II, 1995.

14. Pérez-Calderón, R. Estudio de validación de la metodología para la determinación de vitamina A y E en alimentos infantiles instantáneos por HPLC. Rev Med Exp 2000; 17: 1-4.

15. Mori, T., Beilin, L. Omega-3 fatty acids and inflammation. Curr Atheroscler Rep 2004; 6: 461-7.

16. Crawford, M., Bazinet, R., Sinclair, A. Fat intake and CNS functioning: ageing and disease. Ann Nutr Metab 2009; 55: 202-228.

17. Valenzuela, R., Bascuñán, K., Valenzuela, A. Ácido docosahexaenoico (DHA): una perspectiva nutricional para la prevención de la enfermedad de Alzheimer. Rev Chil Nutr. 2008; 35: 250-61.

18. Calon, F., Colec, G. Neuroprotective action of omega-3 polyunsaturated fatty acids against neurodegenerative diseases: Evidence from animal studies. Prost Leuko Essential Fatty Acids 2007; 77: 287-93.

19. Valenzuela, A. Docosahexaenoic acid (DHA), an essential fatty acid for the proper functioning of neuronal cells: their role in mood disorders. Grasas \& Aceites 2009; 60: 203-12.

20. Smit, L., Mozaffarian, D., Willett, W. Review of fat and fatty acid requirements and criteria for developing dietary guidelines. Ann Nutr Metab 2009; 55: 44-55.

21. Chen, S., Judd, J., Kramer, M., Meijer, G., Clevidence, B., Baer, D. Phytosterol intake and dietary fat reduction are independent and additive in their ability to reduce plasma LDL-cholesterol. Lipids 2009; 44: 273-81.

22. Valenzuela, A., Ronco, AM. Los fitoesteroles: Lípidos de origen natural que protegen la salud cardiovascular. Aceites \& Grasas 2006; XIV: 560-7.

23. Kangmo, L., Mi-Hye, L., Shailendra, P. Dietary Cholesterol absorption; more than just bile. TRENDS Endocrinol Metab 2001; 12: 314-20.

24. Hernández M. Recomendaciones nutricionales para el ser humano: Actualización. Rev Cubana Invest Biomed 2004; 23; 266-92.

25. Ascherio, A. Intake of potassium, magnesium and fibre and the risk of stroke among US men. Circula- 
tion 1998; 98: 1198-204.

26. Bourre, J., Paqueotte, P. Seafood (wild and farmed) for the elderly: Controbution to the dietary intakes of iodine, selenium, DHA and vitamins B12 y D3. J Nutr Health Aging 2008; 12: 186-92.

27. Allen, L. How common is vitamin B-12 deficiency? Am J Clin Nutr 2009; 89: 693S-6S.

28. Brattstrom, L., Wilcken, D. Homocysteine and cardiovascular disease: cause or effect? Am J Clin Nutr 2000; 72: 315-23.
29. Rogovik, A., Vohra, S., Goldman, R. Safety considerations and potential interactions of vitamins: Should vitamins to be considered drugs? Ann Pharmacother 2010; 44: 311-324.

30. Ostlund, R.E. Phytosterols in Human Nutrition. Annu Rev Nutr. 2002; 22:533-49.

31. Cohn, J., Kamili, A., Wat, E., Chung, R., Tandy, S. Reduction in intestinal cholesterol absorption by various food components: Mechanisms and implications. Atherosclerosis 2010: 11; 45-8. 